\title{
Hydrogen sulfide attenuates induction and prevents progress of the 6- hydroxydopamine-induced Parkinsonism in rat through activation of ATP- sensitive potassium channels and suppression of ER stress \\ Azita Minaei, Mohammad Reza Sarookhani, Hashem Haghdoost-Yazdi, Farzad Rajaei \\ Cellular and Molecular Research Center, Research Institute for Prevention of Non-Communicable Disease, Qazvin University of Medical Sciences, Qazvin, Iran
}

\begin{abstract}
Purpose: Studies argue in favor of hydrogen sulfide $\left(\mathrm{H}_{2} \mathrm{~S}\right)$ as the next potent therapeutic agent for neurodegenerative diseases. In present study, we investigated the effect of long term treatment with NaHS (as donor of $\mathrm{H}_{2} \mathrm{~S}$ ) on induction and progress of the 6-hydroxydopamine (6-OHDA) induced Parkinsonism in rat. Methods: The 6-OHDA was injected into medial forebrain bundle of right hemisphere by stereotaxic surgery. Behavioral tests and treatments were carried out to eight weeks after the toxin. Immunohistochemistry and western blotting were carried out to evaluate the survival of tyrosine hydroxylase (TH) -positive neurons in substantia nigra (SN) and also expression of glucose-regulated protein 78 (GRP78) and C/EBP homologous protein (CHOP), the markers of endoplasmic reticulum (ER) stress, in striatum and SN. Results: Eight weeks assessment of the behavioral symptoms showed that NaHS especially at dose of $100 \mu \mathrm{mol} / \mathrm{kg}$ attenuates remarkably induction of the Parkinsonism and prevents its progress. NaHS also increased the survival of TH- positive neurons and suppressed 6-OHDA- induced overexpression of GRP78 and CHOP. Blockade of ATP-sensitive potassium (K-ATP) channels with glibenclamide (Glib) prevented markedly the effect of NaHS on both the induction phase and survival of TH- positive neurons. But Glib did not affect the preventing effect of NaHS on the progress phase and its suppressing effect on the overexpression of ER stress markers. Conclusion: $\mathrm{H}_{2} \mathrm{~S}$ attenuates induction of the 6-OHDA- induced Parkinsonism and also increases the survival of dopaminergic neurons through activation of K-ATP channels. $\mathrm{H}_{2} \mathrm{~S}$ also prevents progress of the Parkinsonism probably through suppression of ER stress. (C) 2021 Elsevier Inc.

keywords

6-hydroxydopamine-induced Parkinsonism; CHOP; GRP78; Hydrogen sulfide; K-ATP channels; TH-positive neurons
\end{abstract}

